\title{
Maternally Inherited Deafness and Unusual Phenotypic Manifestations Associated with A3243G Mitochondrial DNA Mutation
}

\author{
Katalin KOMLÓSI, ${ }^{1}$ Richárd KELLERMAYER, ${ }^{1}$ Anita MAÁSZ, ${ }^{1}$ Viktória HAVASI, ${ }^{1}$ Katalin HOLLÓDY, \\ Olga VINCZE, ${ }^{3}$ Hajnalka MERKLI, ${ }^{4}$ Endre PÁL, ${ }^{4}$ Béla MELEGH ${ }^{1}$
}

\author{
${ }^{1}$ Department of Medical Genetics and Child Development, University of Pécs, ${ }^{2}$ Department of Neurology, \\ Pediatric Clinic, University of Pécs, ${ }^{3}$ Kerpel-Frónius Ödön Children's Hospital of the County Hospital of Baranya, Pécs, \\ ${ }^{4}$ Department of Neurology, University of Pécs, Pécs, Hungary
}

The mitochondrial DNA A3243G transition is a fairly common mutation which often associates with a MELAS (mitochondrial encephalomyopathy, lactic acidosis and stroke-like episodes) phenotype, however, a broad variety in the associated clinical picture has also been described. The patient reported here developed a generalized seizure at age 12, which was followed by bilateral hearing loss and occasional fatigue. The maternal inheritance pattern of hearing loss pointed to a possible mitochondrial origin, which was confirmed by molecular analysis of the mitochondrial DNA, revealing a heteroplasmic A3243G transition. Interestingly, muscle biopsy showed ragged-red fibers in the proband, which is unusual in the deafness-associated forms of this mitochondrial disorder. In addition to hearing impairment in four generations of the family, fatal cerebral embolization in the mother and fatal heart attack in the maternal grandmother (both at age 33) also occurred. On the contrary, diabetes, which usually accompanies the hearing loss variant, was specifically absent in all generations. The unusual manifestations associated with this mutation somewhat differentiate this family from the already known variants. (Pathology Oncology Research Vol 11, No 2, 82-86)

Key words: mtDNA A3243G, tRNA ${ }^{\text {Leu(UUR) }}$, mitochondrial disease, MELAS, deafness

\section{Introduction}

Mitochondrial disorders encompass clinical phenotypes associated with abnormalities of oxidative phosphorylation. Due to the dual genetic control of mitochondria, these diseases can either be caused by primary alterations of the mitochondrial DNA (mtDNA), or by mutations in nuclear genes affecting mitochondrial function. ${ }^{35}$ Important advances have been made regarding the genetic background of mitochondrial disorders in the past decade. ${ }^{7,20}$ Among the diseases affecting mitochondrial tRNA (mt tRNA) genes, two common syndromes are the MELAS syndrome (mitochondrial encephalomyopathy, lactic acidosis and stroke-like episodes), and myoclonic epilepsy with ragged-red fibers syndrome (MERRF) ${ }^{6,23}$ The clini-

Received: Apr 5, 2005; accepted: May 15, 2005

Correspondence: Dr. Béla MELEGH, H-7624 Hungary; Tel./fax: +36-72-536-427, e-mail: Bela.Melegh@aok.pte.hu cal manifestations of mitochondrial diseases are known to be extremely variable. Even the same molecular defect can associate with diverse phenotypes. Progressive hearing loss is often part of the clinical picture in the classic mitochondrial syndromes, while in other disorders hypacusis can be the only sign of a mitochondrial disease. ${ }^{33}$

Maternally inherited hearing loss can be part of a syndrome, or can present as non-syndromic isolated hearing impairment. ${ }^{8}$ In the classic mitochondrial diseases, such as Kearns-Sayre syndrome, MELAS syndrome and MERRF syndrome, hearing loss is often part of the clinical signs. ${ }^{14}$ The maternally inherited diabetes and deafness syndrome (MIDD) is also caused by mtDNA mutations, mostly point mutations affecting mt tRNA genes. In the majority of the cases the same mutation (mtDNA A3243G) is responsible for the disease as for the systemic MELAS syndrome..$^{28,32}$ Non-syndromic hearing loss and ototoxic hearing deficit may also be due to mutations affecting mitochondrial tRNA or rRNA genes. ${ }^{26}$ 
Here we report a 13-year-old boy in whom a mild phenotypic presentation was associated with the A3243G tRNA $^{\text {Leu(UUR) }}$ mutation. The main clinical features were a seizure disorder and mild bilateral sensorineural hearing loss. Early, lethal complications of thrombophilia were present in the maternal family members in addition to sensorineural hearing loss. These are unusual manifestations of this mutation.

\section{Patients and methods}

\section{Case report}

Our patient was born by vaginal delivery, following drug-assisted conception after 13 years of infertility, at week 36 of gestation, with $3050 \mathrm{~g}$. He received exchange blood transfusion in the neonatal period due to Rh incompatibility. His psychomotor development was age-appropriate. At 12 years he suffered a generalized seizure (Grand Mal) with positive EEG (generalized paroxysmal activity). Valproic acid therapy was initiated and he remained asymptomatic. By 13 years, bilateral hearing loss developed. Audiology showed mild sensorineural hearing loss on his right and moderate deficit on his left ear. Besides the hearing loss, he also complained of fatigue, and transient muscular pain. His follow-up EEG recording showed generalized paroxysmal activity, a brain MRI revealed no focal abnormality.

The mother of the proband also suffered from hearing impairment. She died of cerebral embolism shortly after her delivery at the age of 33. The maternal grandmother, who also had hypacusis, suffered a fatal heart attack at the age of 33. The maternal great-grandmother had bilateral deafness as the only symptom.

\section{Mitochondrial DNA analysis}

DNA was extracted from peripheral blood leukocytes using a routine desalting method. The most common mitochondrial DNA point mutations were evaluated in addition to the mtDNA mutations known to be associated with maternally inherited hearing loss (A1555G, A3243G, A7445G, 7472insC, T7511C). The entire mt tRNA ${ }^{\text {Leu(UUR) }}$ gene was also sequenced. This is one of the known mutational hotspots associated with the MELAS syndrome (A3243G, A3252G, G3256T, A3260G, T3271C, T3291C). For amplification and sequencing of the MELAS mutational hotspot the following primers were designed: forward primer, AAA TGA TAT CAT CTC AAC TTA G; reverse primer, GAT GGT GAG AGC TAA GGT C. The PCR amplifications were performed on MJR PTC 200 thermal cyclers with a similar protocol for each mutation investigated. The PCR products were sequenced in both directions with an ABI Prism 3100 Avant Genetic Analyzer using the dye termination technique according to the instructions of the manufacturer (Applied Biosystems, Foster City, CA, USA). For a semiquantitative

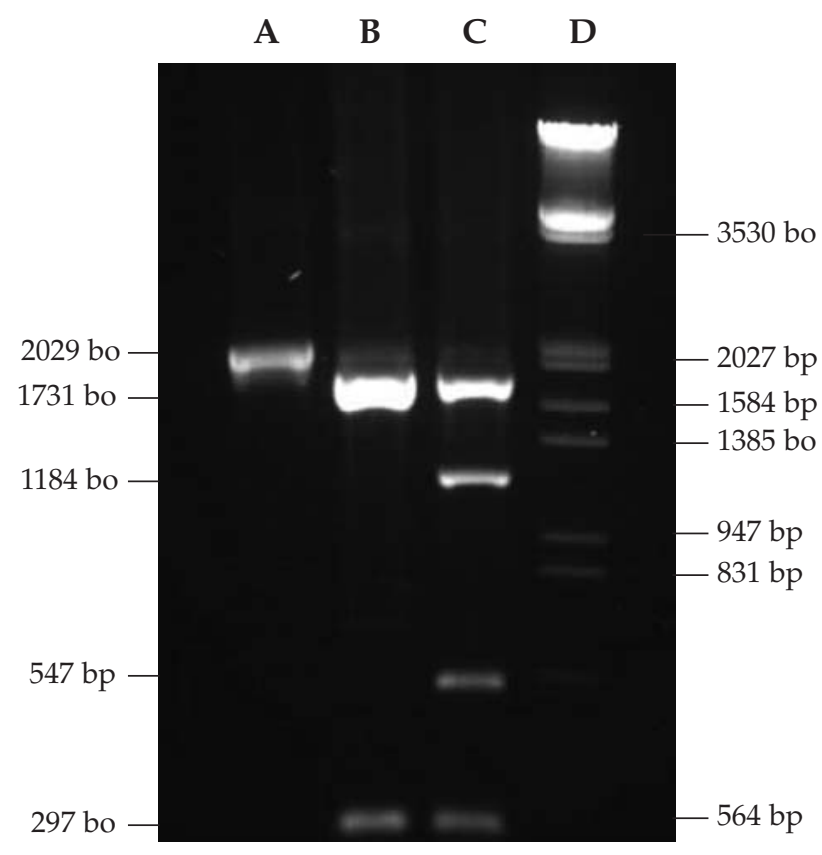

Figure 1. Mutation analysis of the mtDNA at nucleotide position 3243 in the tRNA $A^{\text {Leu(UuR) }}$ gene. Lane A: undigested PCR product (2029 bp). In normal controls, two bands are observed: $1731 \mathrm{bp}$ and $297 \mathrm{bp}$ (Lane B). In our patient two additional cleaved fragments (1184 bp and $547 \mathrm{bp}$ ) appeared after digestion suggesting a heteroplasmic pattern (Lane C). The degree of heteroplasmy was estimated to be $40 \%$ mutant and $60 \%$ wildtype sequence. Lane D: $\lambda$-DNA/ EcoRI+HindIII markers.

analysis of the degree of heteroplasmy a restriction fragment length polymorphism (RFLP) assay was designed. The mtDNA between nucleotides 2695-4724 was amplified by polymerase chain reaction (PCR) and digested with Bsp120I restriction digestion enzyme for the estimation of the degree of heteroplasmy. The wild-type sequence already contains an obligatory Bsp120I cleavage site in order to test the fidelity of the assay. In case of a mutation, replacement of adenine (A) with guanine $(G)$ at nucleotide position 3243 creates a new cleavage site by the restriction enzyme. After digestion the PCR fragments were transferred to a $0.8 \%$ agarose gel and visualized by ethidium bromide stain. The degree of heteroplasmy was estimated according to the intensity of the bands.

Informed consent was obtained from the father and from the patient to all procedures performed.

\section{Results}

The maternal inheritance pattern of hearing impairment prompted a mtDNA evaluation. The mitochondrial DNA analysis from peripheral blood leukocytes of our proband showed a heteroplasmic A-G point mutation at nucleotide position 3243 in the $\mathrm{tRNA}^{\mathrm{Leu}(\mathrm{UUR})}$ gene (Figure 1). The 
analysis of 5 additional known MELAS point mutations in the mt tRNA ${ }^{\text {Leu(UUR) }}$ gene and of the other known point mutations associated with maternally inherited hearing loss gave normal results. During direct sequencing of the mitochondrial $12 \mathrm{~S}$ rRNA of the proband we detected a homoplasmic A-G substitution at nucleotide position 1438, a known polymorphism with no pathological relevance attributed to it so far. During restriction enzyme digestion of the mtDNA fragment containing the mutation, we detected the wild-type sequence in approximately $60 \%$ and the mutated sequence in approximately $40 \%$ (Figure 1).

Parallel to the genetic evaluations, a muscle biopsy was performed to explore his muscular weakness. In the muscle biopsy specimen of our proband (Figure 2) several ragged-red fibers (RRF) could be identified with modified Gömöri trichrome staining (approximately 20\% of the fibers). Succinate dehydrogenase (SDH) staining also showed RRFs, however, strong SDH-reactive blood vessels, characteristic for MELAS, were not detected. The cytochrome oxidase method revealed normal enzyme activity in the majority of the fibers, but cytochrome oxidase-negative ones were found occasionally. The typical features seen in the muscle biopsy specimen supported the presence of mitochondrial myopathy.

Due to the above findings, lactate levels and oral glucose tolerance was tested in the patient. No lactic acidosis, or elevated blood glucose level, nor glycosuria could be detected in repeated evaluations. Additionally, blood glucose and lactate levels were also within normal limits during the oral glucose tolerance test.

The literature suggested that patients with seizures associated with the A3243G mutation may respond adversely to valproic acid. ${ }^{17}$ Consequently, the proband was switched to carbamazepine therapy, but tremors developed. Therefore, lamotrigine was initiated and carbamazepine discontinued. He remains symptom-free on $2 \times 100 \mathrm{mg} /$ day of lamotrigine.

A trial of coenzyme Q10 therapy for 2 months $(3 \times 40$ $\mathrm{mg} /$ day) was also initiated. ${ }^{4,13}$ However, despite of this effort his hearing loss mildly advanced.

\section{Discussion}

According to recent epidemiological studies, mitochondrial disorders are far more frequent than previously believed. Their prevalence is estimated to be $1: 8500 .^{2}$ Thus, they represent a significant group of metabolic diseases. The phenotypic expression of a mitochondrial molecular defect can be influenced by multiple factors, such as the proportion of the mutation in different cells and tissues (the degree of heteroplasmy), and the nuclear genetic background of a given patient. Additionally, mitochondrial DNA haplotype and environmental factors are also known to play a crucial role. ${ }^{8,31,35}$ Consequently, the disorders inherited through the
mtDNA can associate with an extremely variable clinical phenotype. ${ }^{1,22}$ These may remain undiagnosed in case they present with isolated, single symptoms, failing to show the full-blown clinical picture. Among the disorders caused by alterations of the mitochondrial genome, special attention
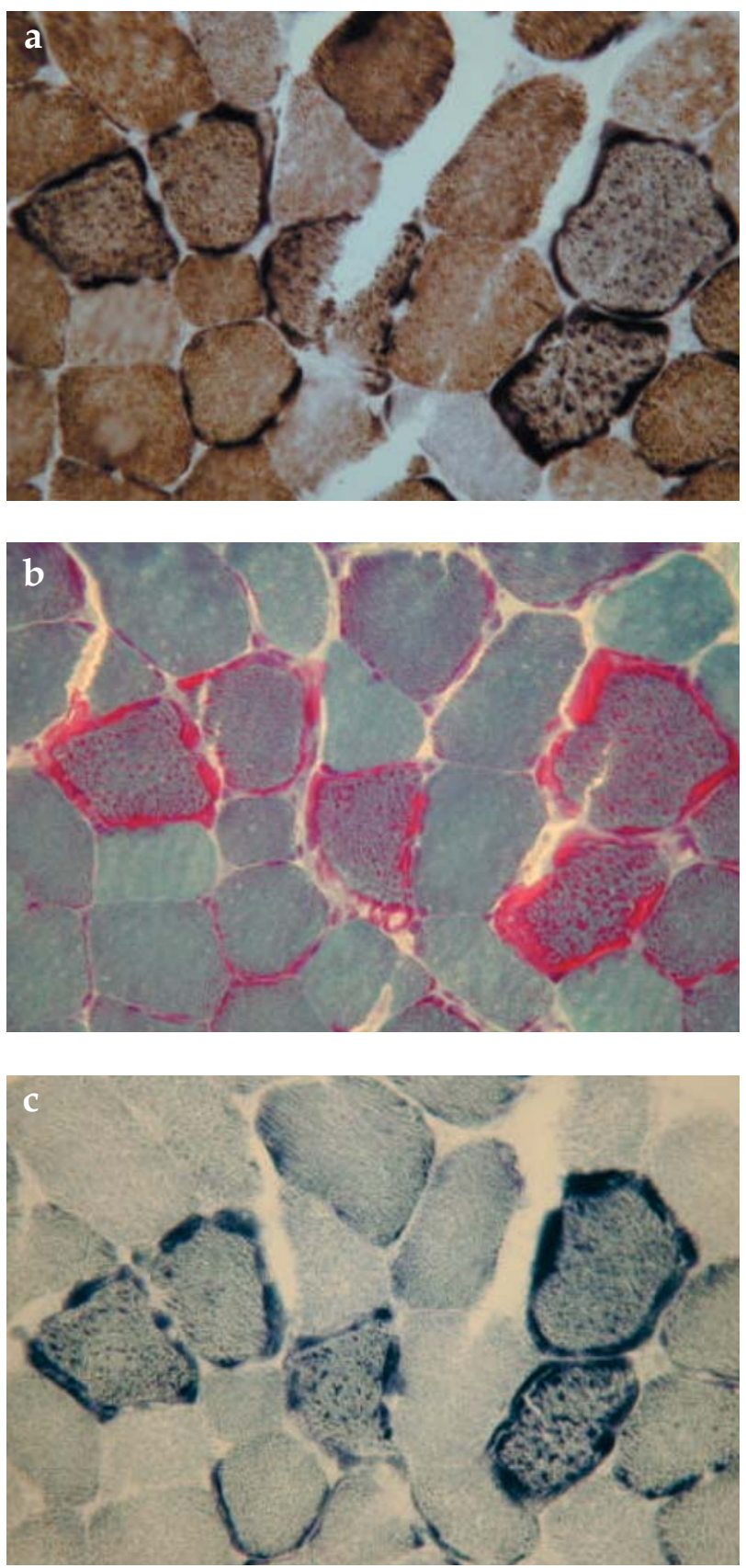

Figure 2. Histochemistry of the muscle biopsy specimen. (a) Cytochrome $c$ oxidase staining: several cytochrome oxidasepositive fibers can be seen. (b) Modified Gömöri trichrome staining revealed approximately $20 \%$ ragged-red fibers. (c) Succinate-dehydrogenase staining $(S D H)$ shows ragged-red fibers, while strong SDH-reactive blood vessels cannot be detected. 
should be given to mitochondrial tRNA gene mutations due to their frequency. A single point mutation affecting a mitochondrial tRNA gene may alter the molecular cellular processes at several different levels: the appropriate mRNA synthesis or translation can be defective, or a mutation influencing the conformation of tRNAs may eventually lead to deficient mitochondrial protein synthesis and, consequently, disturbed oxidative phosphorylation. ${ }^{5}$

The MELAS syndrome is typically a multisystemic disorder presenting in childhood. ${ }^{11,25}$ However, both the appearance of symptoms, as well as the phenotypic spectrum shows a great variety. ${ }^{3,21,27}$ The classic, fully developed clinical picture is dominated by the tonic-clonic seizures, recurrent headaches, episodic vomiting, visual impairment, later the typical stroke-like episodes with transient hemiparesis. Analysis of extended families carrying the A3243G MELAS mutation showed several oligosymptomatic members. In the lack of stroke-like episodes, the most common symptoms were sensorineural hearing loss, ${ }^{8}$ diabetes, ${ }^{9}$ nephropathy, ${ }^{3}$ myopathy, ${ }^{12}$ cardiomyopathy, ${ }^{29}$ cerebellar signs and mental retardation. Among the carriers, asymptomatic members are also known to exist. ${ }^{3}$ Previously, we have reported on the first Hungarian MELAS case with a classic, fully developed phenotype. ${ }^{16}$ Our patient presented here, however, had only one episode of generalized seizure. His main symptom was a slowly deteriorating sensorineural hearing loss. During audiological examination, which showed mild to moderate sensorineural hearing loss, a positive maternal family history was revealed imposing the possibility of mtDNA-inherited hearing impairment. His fatigue and muscle weakness in combination with the hypacusis also pointed to mitochondrial origin.

The A-G transition at np 3243 of the mtDNA affects the tRNA $^{\text {Leu(UUR) }}$ gene. ${ }^{10,15}$ The mutation is observed in a heteroplasmic state in patients. ${ }^{18,19,24}$ In the mtDNA obtained from peripheral leukocytes of our patient, the wild-type sequence was detected in approx. $60 \%$, while the mutated sequence was $40 \%$; there is no data available concerning the degree of heteroplasmy in other tissues. It is known that the $\mathrm{A} 3243 \mathrm{G}$ mtDNA mutation is also associated with the maternally inherited diabetes and deafness syndrome (MIDD). ${ }^{32,28}$ The sensorineural hearing loss associated with this mutation is proposed to be caused by the dysfunction of the stria vascularis and spiral ganglion cells of the inner ear. ${ }^{30}$ According to another examination, the mutation preferentially affects these inner ear structures, and deafness can occur even when the proportion of the A3243G mutation is low. ${ }^{34}$ However, the absence of diabetes and the presence of variable other symptoms in the maternal family members does not correspond to the MIDD syndrome in our case.

Parallel to the molecular genetic studies, a muscle biopsy was performed and the specimen was evaluated for overall histology and histochemistry. Both the modified Gömöri-trichrome staining, and the SDH staining revealed ragged-red fibers (RRF), a hallmark of defective mitochondria. On the other hand, strong SDH-reactive blood vessels, characteristic for MELAS syndrome, could not be detected in the specimen of our patient.

Our patient's final diagnosis and the maternal inheritance pattern of the hearing loss suggest that the audiological deficit in the family was likely due to the mitochondrial DNA A3243G transition. However, the thrombophilic manifestations - such as the fatal stroke-like attack of the mother at the age of 33 and the fatal heart infarct of the grandmother incidentally also at the age of 33 - are not known as usual phenotypic presentations of this mutation. The clinical presentation of our proband and his maternal family members suggest that this known mtDNA defect may associate with unusual phenotypes in the Hungarian population.

Acknowledgement: We are grateful to Judit Oksai and Edit Papp for their excellent technical assistance in the mitochondrial DNA analysis.

\section{References}

1. Bene J, Nádasi E, Kosztolányi G, et al: Congenital cataract as the first symptom of a neuromuscular disease caused by a novel single large-scale mtDNA deletion. Eur J Hum Genet 11: 375379, 2003

2. Chinnery PF, Turnbull DM: Epidemiology and treatment of mitochondrial disorders. Am J Med Genet 106:94-101, 2001

3. Damian MS, Seibel P, Reichmann H, et al: Clinical spectrum of the MELAS mutation in a large pedigree. Acta Neurol Scand 92: 409-415, 1995

4. DiMauro S, Hirano M, Schon EA: Mitochondrial encephalomyopathies: therapeutic approaches. Neurol Sci 21: S901-S908, 2000

5. DiMauro S, Schon EA: Mitochondrial DNA mutations in human disease. Am J Med Genet 106: 18-26, 2001

6. DiMauro S, Hirano M, Kaufmann P, et al: Clinical features and genetics of myoclonic epilepsy with ragged red fibers. Adv Neurol 89: 217-229, 2002

7. DiMauro S, Schon EA: Mitochondrial respiratory-chain diseases. N Engl J Med 348: 2656-2668, 2003

8. Fischel-Ghodsian $N$ : Mitochondrial deafness mutations reviewed. Hum Mut 13: 261-270, 1999

9. Gerbitz KD, van den Ouweland JM, Maassen JA, et al: Mitochondrial diabetes mellitus: a review. Biochim Biophys Acta 1271: 253-260, 1995

10. Goto Y, Nonaka I, Horai S: A mutation in the tRNA(Leu)(UUR) gene associated with the MELAS subgroup of mitochondrial encephalomyopathies. Nature 348: 651-653, 1990

11. Hirano M, Ricci E, Koenigsberger MR, et al: Melas: an original case and clinical criteria for diagnosis. Neuromuscul Disord 2:125-135, 1992

12. Huang CC, Kuo HC, Chu CC, et al: Clinical phenotype, prognosis and mitochondrial DNA mutation load in mitochondrial encephalomyopathies. J Biomed Sci 9: 527-533, 2002

13. Ihara $Y$, Namba $R$, Kuroda $S$, et al: Mitochondrial encephalomyopathy (MELAS): Pathological study and successful therapy with coenzyme $Q_{10}$ and idebenone. J Neurol Sci 90: 263271,1989 
14. Jacobs HT: Mitochondrial deafness. Ann Med 29: 438-491, 1997

15. Kobayashi Y, Momoi MY, Tominaga K, et al: A point mutation in the mitochondrial tRNA(Leu)(UUR) gene in MELAS (mitochondrial myopathy, encephalopathy, lactic acidosis and stroke-like episodes). Biochem Biophys Res Commun 173: 816-822, 1990

16. Komlósi K, Bene J, Havasi V, et al: The A3243G mitochondrial DNA mutation in a Hungarian family (In Hungarian). Orv Heti 35: 1805-1809, 2004

17. Lam CW, Lau CH, Williams JC, et al.: Mitochondrial myopathy, encephalopathy, lactic acidosis and stroke-like episodes (MELAS) triggered by valproate therapy. Eur J Pediatr 156: 562-564, 1997

18. Macmillan C, Lach B, Shoubridge EA: Variable distribution of mutant mitochondrial DNAs (tRNA(Leu[3243])) in tissues of symptomatic relatives with MELAS: the role of mitotic segregation. Neurology 43: 1586-1590, 1993

19. Matthews PM, Hopkin J, Brown RM, et al: Comparison of the relative levels of the $3243(\mathrm{~A} \longrightarrow \mathrm{G})$ mtDNA mutation in heteroplasmic adult and fetal tissues. J Med Genet 31: 41-44, 1994

20. McFarland R, Taylor RW, Turnbull DM: The neurology of mitochondrial DNA disease. Lancet Neurol 1: 343-351, 2002

21. Montagna $P$, Gallassi $R$, Medori $R$, et al: MELAS syndrome: characteristic migrainous and epileptic features and maternal transmission. Neurology 38: 751-754, 1998

22. Munnich A, Rustin P: Clinical spectrum and diagnosis of mitochondrial disorders. Am J Med Genet 106: 4-17, 2001

23. Oldfors A, Tulinius $M$ : Mitochondrial encephalomyopathies. J Neuropathol Exp Neurol 62: 217-227, 2003

24. Ozawa M, Nonaka I, Goto Y: Single muscle fiber analysis in patients with 3243 mutation in mitochondrial DNA: comparison with the phenotype and the proportion of mutant genome. J Neurol Sci 159: 170-175, 1998

25. Pavlakis SG, Phillips PC, DiMauro S, et al: Mitochondrial myopathy, encephalopathy, lactic acidosis, and strokelike episodes: a distinctive clinical syndrome. Ann Neurol 16: 481488, 1984

26. Prezant TR, Agapian JV, Bohlman MC, et al: Mitochondrial ribosomal RNA mutation associated with both antibioticinduced and non-syndromic deafness. Nat Genet 4: 289-294, 1993

27. Pronicki M, Sykut-Cegielska J, Mierzewska H, et al: Diversity of clinical symptoms in A3243G mitochondrial DNA mutation (MELAS syndrome mutation). Med Sci Monit 8: CR767CR773, 2002

28. Reardon W, Ross RJM, Sweeney $M G$, et al: Diabetes mellitus associated with a pathogenic point mutation in mitochondrial DNA. Lancet 340: 1376-1379, 1992

29. Silvestri G, Bertini E, Servidei S, et al: Maternally inherited cardiomyopathy: a new phenotype associated with the A to $G$ AT nt.3243 of mitochondrial DNA (MELAS mutation). Muscle Nerve 20: 221-225, 1997

30. Takahashi K, Merchant SN, Miyazawa T, et al: Temporal bone histopathology and quantitative analysis of mitochondrial DNA in MELAS. Laryngoscope 113: 1362-1368, 2003

31. Thorburn DR, Dahl HH: Mitochondrial disorders: genetics, counseling, prenatal diagnosis and reproductive options. Am J Med Genet 106: 102-114, 2001

32. van den Ouweland JMW, Lemkes HHPJ, Ruitenbeek W, et al: Mutation in mitochondrial tRNALeu (UUR gene in a large pedigree with maternally transmitted Type II diabetes mellitus and deafness. Nat Genet 1: 368-371, 1992

33. Willems PJ: Genetic causes of hearing loss. N Engl J Med 342: 1101-1109, 2000

34. Yamasoba T, Tsukuda K, Oka Y, et al: Cochlear histopathology associated with mitochondrial transfer RNA(Leu(UUR)) gene mutation. Neurology 52: 1705-1707, 1999

35. Zeviani $M$, Spinazzola A, Carelli $V$ : Nuclear genes in mitochondrial disorders. Curr Opin Genet Dev 13: 262-270, 2003 